\title{
Erratum to: The year-long unprecedented European heat and drought of 1540 - a worst case
}

\author{
Oliver Wetter • Christian Pfister - Johannes P. Werner • \\ Eduardo Zorita • Sebastian Wagner • \\ Sonia I. Seneviratne • Jürgen Herget • Uwe Grünewald • \\ Jürg Luterbacher • Maria-Joao Alcoforado • \\ Mariano Barriendos • Ursula Bieber • Rudolf Brázdil • \\ Karl H. Burmeister • Chantal Camenisch • \\ Antonio Contino • Petr Dobrovolný • Rüdiger Glaser • \\ Iso Himmelsbach • Andrea Kiss • Oldřich Kotyza • \\ Thomas Labbé • Danuta Limanówka • \\ Laurent Litzenburger • Øyvind Nordli • Kathleen Pribyl • \\ Dag Retsö • Dirk Riemann • Christian Rohr • \\ Werner Siegfried • Johan Söderberg • \\ Jean-Laurent Spring
}

Published online: 11 July 2014

(C) Springer Science+Business Media Dordrecht 2014

\section{Erratum to: Climatic Change \\ DOI 10.1007/s10584-014-1184-2}

Unfortunately, a mistake was introduced in the family name of Øyvind Nordli during the editorial process. In the original publication of this article it is incorrectly displayed as Nordl instead of Nordli.

Furthermore, we wish to point out that Giessen in the affiliation of J.P. Werner and J. Luterbacher, and Cottbus in the affiliation of U. Grünewald are both situated in Germany rather than Switzerland.

The online version of the original article can be found at http://dx.doi.org/10.1007/s10584-014-1184-2.

O. Wetter $(\bowtie) \cdot$ C. Pfister $(\bowtie) \cdot$ C. Camenisch $\cdot$ C. Rohr

Oeschger Centre for Climate Change Research, University of Bern, Bern, Switzerland

e-mail: oliver.wetter@hist.unibe.ch

e-mail: christian.pfister@hist.unibe.ch

O. Wetter $\cdot$ C. Camenisch $\cdot$ C. Rohr

Institute of History, Section of Economic, Social and Environmental History (WSU), University of Bern, Erlachstrasse 9a, Bern 9 3000, Switzerland

J. P. Werner $(\bowtie) \cdot J$. Luterbacher

Department of Geography; Climatology, Climate Dynamics and Climate Change, Justus Liebig University of Giessen, Senckenbergstrasse 1, 35390 Giessen, Germany

e-mail: johannes.werner@geogr.uni-giessen.de

J. Luterbacher

e-mail: juerg.luterbacher@geogr.uni-giessen.de 
E. Zorita $\cdot$ S. Wagner

Institute of Coastal Research, Helmholtz Centre Geesthacht, Max-Planck-Strasse 1, 21502 Geesthacht, Germany

\section{E. Zorita}

e-mail: eduardo.zorita@hzg.de

\section{S. I. Seneviratne}

Institute for Atmospheric and Climate Science, ETH Zurich, CHN N11, Universitätstrasse 16, 8092 Zurich, Switzerland

e-mail: sonia.seneviratne@env.ethz.ch

J. Herget

Department of Geography, Rheinische Friedrich Wilhelms University, Meckenheimer Allee 166,

D-53115 Bonn, Germany

e-mail: herget@giub.uni-bonn.de

U. Grünewald

Chair Hydrology and Water Resources Management, Faculty of Environmental Sciences and Process

Engineering, Brandenburg University of Technology Cottbus - Senftenberg, Konrad-Wachsmann-Allee 6, 03046 Cottbus, Germany

e-mail: uwe.gruenewald@tu-cottbus.de

\section{M.-J. Alcoforado}

Centre of Geographical Studies, Institute of Geography and Planning, University of Lisbon, Edifício da Fac. de Letras, Alameda da Universidade, 1600-214 Lisboa, Portugal

e-mail: mjalcoforado@campus.ul.pt

M. Barriendos

Catalan Institute for Climate Sciences (IC3), Doctor Trueta 203, 08005 Barcelona, Spain

e-mail: mbarriendos@ub.edu

M. Barriendos

Department of Modern History, University of Barcelona, Montalegre 6, 08003 Barcelona, Spain

U. Bieber

Department of Slavonic Studies and Interdisciplinary Centre of Medieval Studies, University of Salzburg, Erzabt-Klotz-Strasse 1, 5020 Salzburg, Austria

e-mail: ursula.bieber@sbg.ac.at

R. Brázdil • P. Dobrovolný

Institute of Geography, Masaryk University, Kotlářská 2, 61137 Brno, Czech Republic

R. Brázdil

e-mail: brazdil@sci.muni.cz

R. Brázdil • P. Dobrovolný

Global Change Research Centre AS CR, Bĕlidla 956/4a, 60300 Brno, Czech Republic

K. H. Burmeister

Hoyerberg, Am Staeuben 18, Hoyerberg, 88131 Bodolz, Germany

e-mail: k.h.a.burmeister@web.de

A. Contino

Department of Earth and Sea Sciences (DiSTeM), University of Palermo, Via Archirafi 26, 90123 Palermo, Italy

e-mail: ntncontino@gmail.com 
R. Glaser · I. Himmelsbach • D. Riemann

Department of Environmental Social Studies and Geography - Physical Geography, Albert-Ludwigs University Freiburg i.Br., Werthmannstrasse 4, 79102 Freiburg, Germany

R. Glaser

e-mail: ruediger.glaser@geographie.uni-freiburg.de

\section{A. Kiss}

Institute of Hydraulic Engineering and Water Resources Management, Vienna University of Technology, Karlsplatz 13, 1040 Vienna, Austria

e-mail: kiss@hydro.tuwien.ac.at

\section{O. Kotyza}

Regional Museum, Dlouhá 139, 41201 Litoměřice, Czech Republic

e-mail: muzeum.1tm@telecom.cz

T. Labbé

Institute of History, Technische Universität (TU) of Darmstadt, Residenzschloss, 64283 Darmstadt, Germany

e-mail: labbe@pg.tu-darmstadt.de

D. Limanówka

Institute of Meteorology and Water Management National Research Institute (IMGW-PIB), Center for Poland's Climate Monitoring, Podleśna, 01-673 Warsaw, Poland

e-mail: danuta.limanowka@imgw.pl

L. Litzenburger

Lorraine University Center for Historical Research (CRULH), University of Lorraine, Campus Lettres et Sciences Humaines, Place Godefroy de Bouillon, BP 3397, 54015 Nancy Cedex, France e-mail: 1.litzenburger@free.fr

Ø. Nordli

Norwegian Meteorological Institute, Research and Development Department, Division for Model and Climate Analysis, Henrik Mohns plass 1, P.O. Box 43, Blindern N-0313, Norway e-mail: oyvind.nordli@met.no

K. Pribyl

Climatic Research Unit, School of Environmental Sciences, University of East Anglia, Norwich Research Park, Norwich NR4 7TJ, UK

e-mail: k.pribyl@uea.ac.uk

D. Retsö · J. Söderberg

Department of Economic History, Stockholm University, SE-106 91 Stockholm, Sweden

D. Retsö

e-mail: dag.retso@lai.su.se

J. Söderberg

e-mail: johan.soderberg@ekohist.su.se

\section{W. Siegfried}

Agroscope Research Station ACW, Extension Wine, Schloss 1 Postfach, 8820 Wädenswil, Switzerland e-mail: werner.siegfried@acw.admin.ch

\section{J.-L. Spring}

Station de recherche Agroscope à Pully, 21 av. de Rochettaz, CH-1009 Pully, Switzerland

e-mail: jean-laurent.spring@agroscope.admin.ch 